\title{
A Systematic Analysis of Real-World Energy Blockchain Initiatives
}

\author{
Peter O'Donovan *(D) and Dominic T. J. O'Sullivan \\ IERG, University College Cork, T12 K8AF Cork, Ireland \\ * Correspondence: peter_odonovan@umail.ucc.ie
}

Received: 12 July 2019; Accepted: 6 August 2019; Published: 10 August 2019

\begin{abstract}
The application of blockchain technology to the energy sector promises to derive new operating models focused on local generation and sustainable practices, which are driven by peer-to-peer collaboration and community engagement. However, real-world energy blockchains differ from typical blockchain networks insofar as they must interoperate with grid infrastructure, adhere to energy regulations, and embody engineering principles. Naturally, these additional dimensions make real-world energy blockchains highly dependent on the participation of grid operators, engineers, and energy providers. Although much theoretical and proof-of-concept research has been published on energy blockchains, this research aims to establish a lens on real-world projects and implementations that may inform the alignment of academic and industry research agendas. This research classifies 131 real-world energy blockchain initiatives to develop an understanding of how blockchains are being applied to the energy domain, what type of failure rates can be observed from recently reported initiatives, and what level of technical and theoretical details are reported for real-world deployments. The results presented from the systematic analysis highlight that real-world energy blockchains are (a) growing exponentially year-on-year, (b) producing relatively low failure/drop-off rates ( $7 \%$ since 2015$)$, and (c) demonstrating information sharing protocols that produce content with insufficient technical and theoretical depth.
\end{abstract}

Keywords: energy; blockchain; smart grid; microgrid

\section{Introduction}

An energy blockchain may be considered a distributed ledger dedicated to the management of energy transactions between generation and load nodes across power systems [1]. These ledgers promise to significantly disrupt the energy sector by lowering the cost of economic transactions and removing superfluous third parties from the energy value chain. However, large-scale and grid-integrated energy blockchains needed to facilitate real-world operations are commonly owned by private entities with a strong commercial focus, which can naturally impede the dissemination of technical specifications, energy market models and engineering knowledge to the wider research community. Although innovative and important research can be undertaken within small-scale experimental environments, research efforts focused on developing high-impact and commercial-oriented energy blockchains should integrate and interoperate with existing private and public infrastructure [2]. This includes the establishment of information streams between physical metering and energy blockchains, which has been highlighted as an important challenge for system regulators and energy providers [3]. Indeed, blockchains cannot be operationalised in real-world energy operations without sufficient cooperation between energy providers, grid operators and government agencies [4].

At present, applied academic and industrial researchers wishing to advance the development and adoption of real-world energy blockchains may be impeded due to limited visibility of methods and challenges. This research aims to explore and understand the current state of real-world energy 
blockchains, and highlight prominent trends relating to growth rates, failure rates, information sharing and technology adoption. Some of the primary challenges specific to real-world energy blockchains are summarised below.

- Infrastructure and environment: energy providers and grid operators control the infrastructure needed for large-scale integrated energy blockchains. Therefore, applied researchers are somewhat restricted to theoretical and experimental contributions that may not directly align with real-world environments.

- Technical transparency: blockchain platforms and smart contracts are highly configurable technologies, which can be designed using many different frameworks, architectures and algorithms. However, technical details from real-world energy blockchain pilots(e.g., blockchain platform and consensus algorithm) are difficult to acquire, while negative outcomes and technical failures (e.g., approaches that did not scale) are not reported.

- Project innovation velocity: given the contemporary and experimental nature of energy blockchains, strategic direction and technical approaches may change rapidly using quick and iterative development cycles. Although such changes are expected, the reasons and insights driving change and innovation are not reported to the broader researcher community. Of course, while game-changing insights may represent key competitive advantages that cannot be publicly shared, exposing less commercially sensitive insights from across industry could prove an invaluable resource to the field.

\section{Background}

\subsection{Energy Blockchain Applications}

The most prominent energy blockchain applications include peer-to-peer trading, electric vehicle charging, sustainability rewards, prepaid metering, energy performance, and demand-side management, to name a few [4]. Of these applications, peer-to-peer and transactive energy trading for smart grids and microgrids represent some of the most compelling and researched use cases, with research contributions focusing on efficient and robust designs to facilitate energy transactions across communities and wholesale markets [5-9].

Many blockchain architectures and smart contracts have been proposed to enable transactive energy trading, auction management and security within smart grids $[10,11]$, while continuous double auction mechanisms have also been considered for microgrids [12]. In order to transition theoretical and experimental blockchain architectures to real-world environments, the identification and integration of engineering principles and market dynamics must also be considered. Examples of energy-specific requirements may include the ability to track and attribute energy losses across peer-to-peer transactions [1], automate the negotiation, settlement and payment of pre-time-of-use market pricing [3], or reward customers for demand-side management participation [3].

Generally, early energy blockchain ideologies focused on the disintermediation of residential customers from traditional energy providers. However, energy blockchains can also deliver value and efficiencies for commercial and industrial customers. For example, energy blockchains and smart contracts have been proposed to manage energy performance contracts between clients and service providers, whereby measured data, analytics models and contractual obligations are stored within an immutable ledger to promote trust between stakeholders [2]. Another compelling set of energy applications are emerging around vehicle-to-grid integrations and services, where the adoption of blockchain technologies and smart contracts can be used to develop decentralised applications for (a) identifying charging stations based on journey routing, battery status and current traffic conditions, and (b) settling payments at charging stations [13]. 


\subsection{Potential Benefits}

The most obvious benefit of real-world energy blockchains is the democratisation of energy transactions, whereby blockchain technology may be used to remove intermediaries from the energy value chain and enable communities to execute transactions independently. These independent two-way markets should provide market participants (e.g., customers) with sufficient transaction transparency, security and reliability, which can be satisfied using the blockchain's immutable and decentralised architecture $[10,12,14,15]$. Additionally, energy blockchains can reduce transaction costs and settlement times [10], substitute intermediaries with consensus models [10], and increase profitability and minimise costs for participants using sophisticated market intelligence (e.g., machine learning) [12].

\subsection{Challenges and Motivation}

There are many potential technical and market challenges that must be considered to advance the development and adoption of real-world energy blockchains. Some of the more prominent and obvious challenges are summarised below.

- Regulation will be needed to support the introduction of co-operatives and private individuals across the energy value chain [16], given new types of market participants (e.g., peer-to-peer prosumers) cannot realistically adhere to the same regulations as large-scale energy providers [17].

- Scalability concerns relating to different aspects of energy blockchains must be addressed to enable large-scale rollouts on public infrastructure [4]. A consequence of poor scalability may manifest unreliable real-time transaction performance, and unsustainable blockchains that consume more energy than the transactive value of underlying energy trades [14]. These economically imbalanced blockchains are somewhat synonymous with the proof-of-work consensus model, where computationally intensive cryptographic problems must be solved to commit transactions. Additionally, other scalability concerns pertaining to memory and compute limitations may arise when advanced models (e.g., machine learning) and ancillary operating data are embedded within the blockchain [2]. Therefore, engineers must carefully consider which assets belong inside and outside the blockchain to promote scalability [3,5].

- Integration methods for (a) blockchain-to-grid and (b) blockchain-to-blockchain must be addressed to ensure real-time, reliable and trusted information flows between market participants and stakeholders. Interfaces with a physical grid infrastructure should be considered important when financially accounting for two-way electricity flows, dynamic tariffs and market models [13], while integration and interoperability between disparate energy blockchains may be needed, where energy providers and grid operators support different architectures and topologies [4].

- Market policies and logic are needed to ensure the trust and security characteristics of blockchain technology are not circumvented by ambiguous transactional data, or inaccurate business logic. Such market challenges may include the development of formal methods to identify, calculate and settle energy losses between different combinations of market participants [5], and the introduction of formal and trusted methods to manage the temporal lifecycle of smart contracts as terms and conditions change [2].

\section{Methodology}

The following sections describe the research process used to explore real-world energy blockchain initiatives reported in the literature. Figure 1 illustrates the research process workflow as three distinct layers-(1) the top layer focuses on identifying published peer-reviewed systematic studies and extracting the reported real-world applications, (2) the middle layer focuses on reviewing the extracted projects and applying inclusion/exclusion criteria to ensure only real-world applications are evaluated, and (3) the bottom layer focuses on generating metadata about the real-world applications to visualise and analyse trends. 


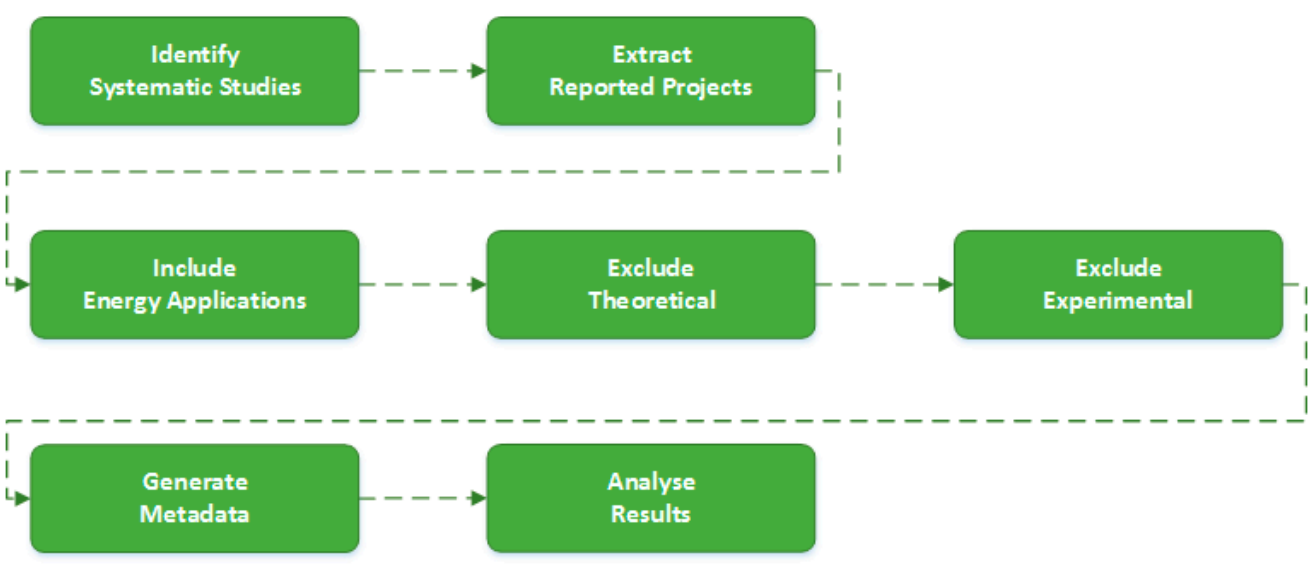

Figure 1. Data extraction and analysis process.

\subsection{Research Objectives}

The overarching and fundamental aim of this research was to explore and understand the current state of real-world energy blockchains. Table 1 shows the specific research objectives and dimensions that were chosen to characterise and comprehend these real-world initiatives.

Table 1. Guiding research objectives and rationale.

\begin{tabular}{ccc}
\hline ID & Objective & Rationale \\
\hline RO1 & $\begin{array}{c}\text { Determine growth } \\
\text { rates and trends }\end{array}$ & $\begin{array}{c}\text { Understanding the current growth rates can be used to infer interest in } \\
\text { the field, while high growth rates should create some urgency around } \\
\text { the establishment of structured research agendas and themes. }\end{array}$ \\
\hline RO2 & $\begin{array}{c}\text { Determine failure rates } \\
\text { and trends }\end{array}$ & $\begin{array}{c}\text { Understanding the failure rates can be used to infer poor business cases } \\
\text { or technology choices, while sharing reasons for failures could serve to } \\
\text { shape future research efforts and best-practices. }\end{array}$ \\
RO3 & $\begin{array}{c}\text { Determine method of } \\
\text { sharing information }\end{array}$ & $\begin{array}{c}\text { Understanding how energy blockchain initiatives disseminate } \\
\text { information could indicate the scale of the initiative, or level of } \\
\text { information sharing. }\end{array}$ \\
\hline RO4 & $\begin{array}{c}\text { Determine depth of } \\
\text { technical information }\end{array}$ & $\begin{array}{c}\text { Understanding the level of information sharing currently observed } \\
\text { across energy blockchain initiatives could serve to highlight a } \\
\text { significant barrier impeding research and industry alignment. }\end{array}$ \\
\hline RO5 & $\begin{array}{c}\text { Determine technical } \\
\text { trends }\end{array}$ & $\begin{array}{c}\text { Understanding the energy applications, blockchain platforms and } \\
\text { consensus algorithms provides insights regarding current approaches } \\
\text { and trends. }\end{array}$ \\
\hline
\end{tabular}

\subsection{Search Strategy and Scope}

The search strategy focused on identifying peer-reviewed secondary research that employed systematic methods to investigate energy blockchains, whereby the reported energy blockchain initiatives could be extracted for analysis. Table 2 shows the terms and logical conditions used to search for publications within prominent repositories, including Science Direct, Scopus, Google Scholar, and IEEE Xplore. Given the research approach focused specifically on identifying systematic studies, the term 'Systematic' was mandatory, while some flexibility was given to allow for variations in the publication's title-using 'Review', 'Mapping', 'Study' or 'Survey' yielded results where any of these terms appeared in the title along with 'Systematic' (e.g., Systematic Review, Systematic Mapping, etc.). After using the terminology to search each repository, only one systematic study on energy blockchains was identified [18]. However, other systematic studies made reference to energy applications within a broader scope of work $[19,20]$. 
Table 2. Search terminology and logical conditions.

\begin{tabular}{cc}
\hline Search Terminology & Logical Condition \\
\hline Systematic & AND \\
(Review OR Mapping OR Study OR Survey) & AND \\
Energy & AND \\
Blockchain & \\
\hline
\end{tabular}

Choosing to target studies adhering to systematic approaches was deemed appropriate given the method could facilitate broad data collection (i.e., identify projects), remove aspects of researcher bias (e.g., search preferences), and present concise tabular data. Although building a new dataset from online searches was considered, the identified systematic review was of (a) high-quality, (b) peer-reviewed, and (c) recently published. Therefore, the benefit of conducting an unbounded internet search for additional energy blockchain initiatives was unnecessary.

\subsection{Filtering, Extraction and Analysis}

After identifying energy blockchain initiatives from the existing study, several inclusion and exclusion criteria were applied to remove some initiatives from the analysis. The intention of the chosen criteria was to emphasise real-world initiatives. First, only projects focused exclusively on energy applications (e.g., peer-to-peer trading) were included. Second, early-stage theoretical projects (e.g., architecture, design, etc.) were excluded. Finally, experimental projects without real-world origins (e.g., no interaction with grid or infrastructure) were also excluded.

After inclusion/exclusion criteria were applied, each initiative was reviewed to identify and extract data points needed for analysis. Table 3 describes the data points within the dataset-the original data (e.g., name, platform, country, etc.) was augmented with additional data (e.g., activity status) to produce a dataset aligned with the objectives of this research, and facilitate the identification of prominent trends and patterns.

Table 3. Data extraction for energy blockchain projects.

\begin{tabular}{|c|c|}
\hline Data & Reasoning \\
\hline Country & Highlight clusters of energy blockchain investment and innovation. \\
\hline Year & $\begin{array}{l}\begin{array}{l}\text { Establish temporal data to illustrate the popularity and prevalence of energy } \\
\text { blockchain projects. }\end{array}\end{array}$ \\
\hline Project Name & $\begin{array}{l}\text { Differentiate between multiple energy blockchain initiatives undertaken within } \\
\text { the same organisation. }\end{array}$ \\
\hline Current Status & Identify active and inactive projects to establish failure rates and project lifetimes. \\
\hline Project URL & Access the most up to date information relating to the project. \\
\hline Field of Activity & Understand the energy applications and use cases driving adoption. \\
\hline Information Sharing & $\begin{array}{l}\text { Highlight the level of technical and theoretical details accessible to the broader } \\
\text { research community. }\end{array}$ \\
\hline Controls Dissemination & $\begin{array}{l}\text { Indicate whether information sharing and dissemination is controlled by the } \\
\text { primary stakeholders, or third parties. }\end{array}$ \\
\hline Blockchain Platform & $\begin{array}{l}\text { Determine the most prominent blockchain technology used to implement } \\
\text { real-world energy blockchains. }\end{array}$ \\
\hline \multirow[t]{2}{*}{ Consensus Algorithm } & $\begin{array}{l}\text { Determine the most prominent consensus algorithms used to implement } \\
\text { real-world energy blockchains. }\end{array}$ \\
\hline & $\begin{array}{l}\text { Identify real-world energy blockchain applications with substantive case studies } \\
\text { that have been subjected to the peer-review process. }\end{array}$ \\
\hline Research Project & Determine whether the initiative originated from academic or commercial entities. \\
\hline
\end{tabular}




\subsection{Threats and Limitations}

There are many inherent threats and limitations associated with systematic approaches that relate to data collection, information extraction and data analysis. The primary threats and limitations associated with this research are summarised below;

- Relevance of identified projects: this research uses energy blockchain initiatives from an existing systematic study, which means initiatives omitted from the original study shall be excluded from the analysis and results presented. However, given the original study was peer-reviewed, one can reasonably assume that (a) prominent projects and initiatives were captured, and (b) unbiased selection methods were used to identify initiatives.

- Project selection and filtering: the criteria chosen for filtering energy blockchain initiatives were derived solely from discussions between researchers, which naturally introduces the possibility of biases influencing the final dataset. However, such biases were largely mitigated using top-level and generic criteria to isolate energy blockchain initiatives that were associated with real-world cases. Indeed, only nine initiatives were filtered from the 140 initiatives presented in the original study.

- Soft and inconsistent data: superficial reporting of commercial real-world energy blockchain initiatives may affect the robustness of the final dataset. In particular, insufficient technology, engineering and business details could lead to misclassified characteristics. Although not realistic to completely mitigate data quality issues stemming from superficial reporting, encountering quality concerns served to highlight the need for improved information sharing and reporting to progress the research agenda.

\section{Results and Discussion}

The following section presents results and discussions from the analysis of real-world energy blockchain initiatives, which was undertaken to align with the specified research objectives. The final dataset comprised 131 energy blockchain initiatives, of which there was only one with strong links to academic research, and one peer-reviewed publication. Thus, the initiatives analysed may be considered well-aligned with the perspective of this research, which focuses on evaluating the type of real-world initiatives being undertaken, and determining to what extent information from these initiatives can inform academic and industrial research efforts.

\subsection{RO1: Determine Growth Rates and Trends}

Figure 2 illustrates energy blockchain initiatives formed between 2014 and 2018. The analysis shows exponential growth was experienced between 2015 and 2017, with a lower number of reported initiatives in 2018 due to data collection being completed circa Q1 2018. Assuming energy blockchains continue to experience strong and positive growth over the next number of years, standardising methods, technologies and approaches must be considered an important requirement to ensure reasonable levels of commonality, and discourage ad hoc proprietary implementations that may prove difficult to change retrospectively. 


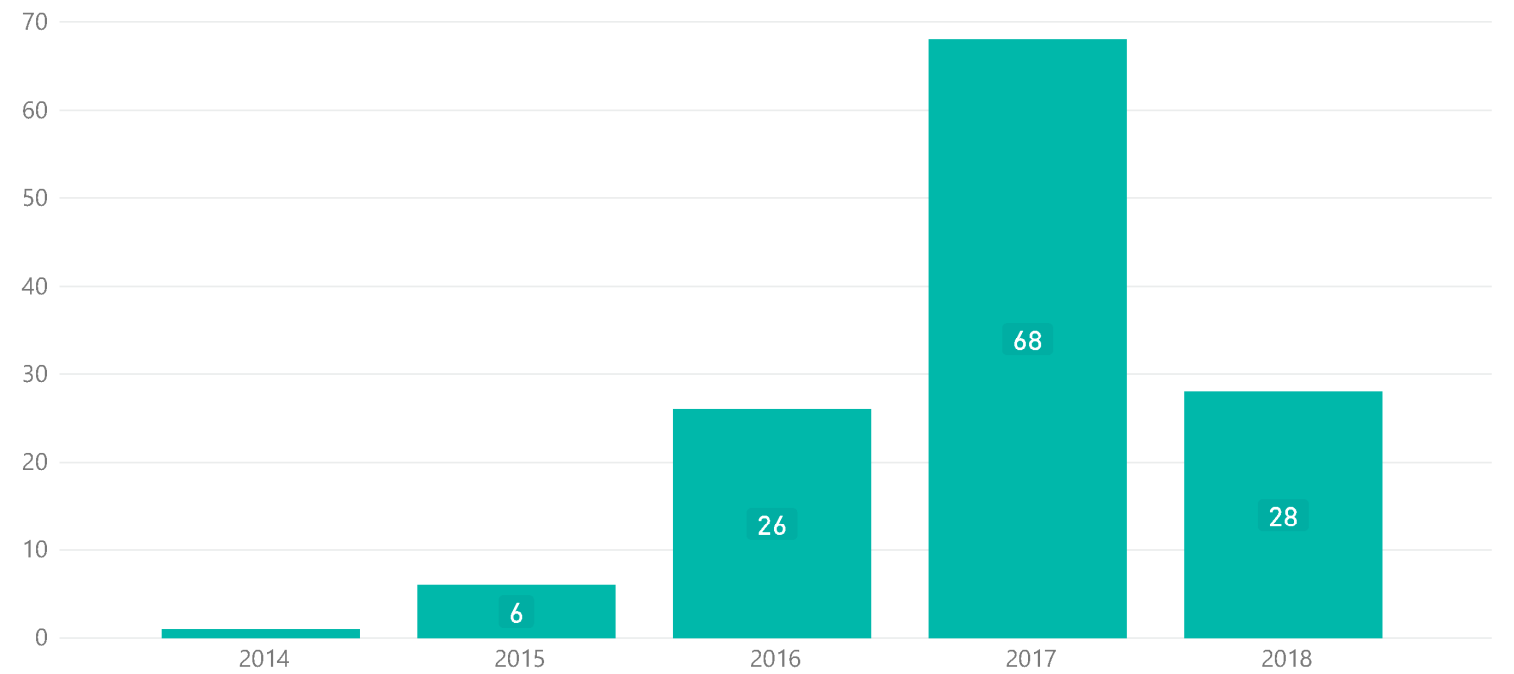

Figure 2. Energy blockchain initiatives by year.

Capturing each initiative by year was the only variable needed to determine basic annual growth and trends pertaining to energy blockchains. However, multiple data sources and subjective decision-making were needed to populate the year variable depending on the type of organisation and initiative. For example, blockchain solution providers have company formation dates (e.g., CrunchBase), public cryptocurrencies and initiatives have initial coin offering dates, and energy providers have press releases promoting pilots and projects. Some of the main weaknesses observed during the data collection process included (a) inconsistent and conflicting formation dates, and (b) poor visibility and confidence relating to data integrity.

\subsection{RO2: Determine Failure Rates and Trends}

Figure 3 illustrates the percentage of active and inactive energy blockchain initiatives that exist based on their formation year. Given there was no single data point or information source to determine the status of energy blockchains, status was derived using the date of the most recent public update, whereby those disseminating information (e.g., blog, press release, report, whitepaper) within the last 12 months were deemed active. Interestingly, this shows the majority of reported energy blockchain initiatives remain active, which would seem counterintuitive when considering the contemporary and experimental nature of blockchain technology. Although quite difficult to prove without transparent reporting and visibility, one possible reason for low failure and decommissioning rates may stem from organisations extending the lifetime of initiatives by pivoting and evolving different concepts, rather than creating new initiatives. 


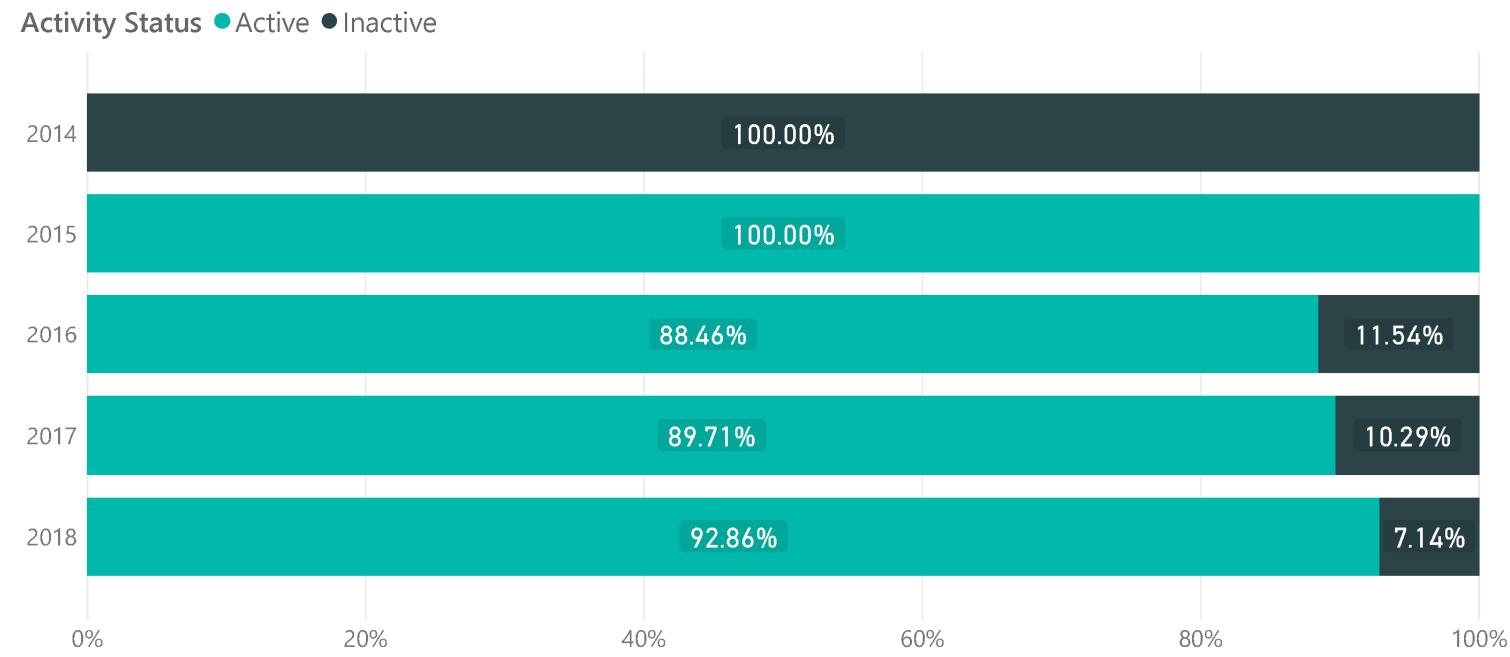

Figure 3. Estimated status based on most recent updates.

Ideally, the status of energy blockchains could be objectively determined using some trusted source. However, the intertangled and disparate nature of real-world energy blockchains present non-trivial reporting challenges. Thus, deriving status using publicly accessible and timestamped sources of information would appear a reasonable solution for high-level analysis that can tolerate minor deviations. Of course, when using such an approach, one cannot say with absolute certainty that initiatives reported during the previous 12 months are genuinely active and operational blockchains. This type of uncertainty further highlights the need for standard, consistent and transparent reporting of real-world energy blockchains that can be utilised to establish accurate trends and patterns.

\subsection{RO3: Determine Method of Sharing Information}

Figure 4 illustrates the distribution of communication channels (e.g., project websites, industry portals etc.) that are owned versus those of third parties, where information about the initiative has been shared. Identifying ownership of communication channels proved useful to broadly differentiate between large-scale projects and small-scale pilots, which was based on the assumption that large-scale real-world projects may need to inform customers, investors or other stakeholders of progress, etc. Naturally, these dedicated communication channels also offered the potential to provide more insights relating to the initiative using blogs, newsfeeds and technical whitepapers. The analysis shows the majority of blockchain initiatives targeting prominent energy applications (e.g., energy trading, investments and asset management) own and operate dedicated communication channels (e.g., websites), with ownership ranging from $65 \%$ to $77 \%$ across the top three energy applications. 


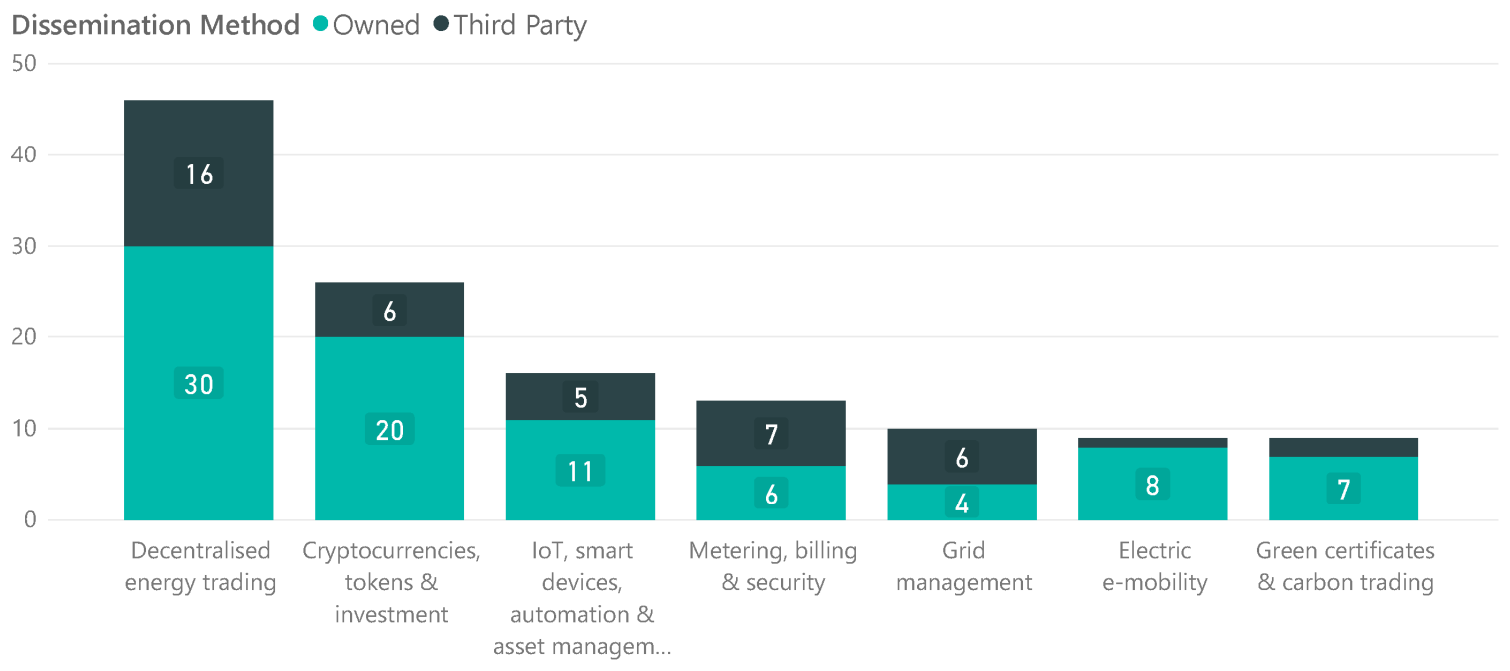

Figure 4. Dissemination methods for energy blockchain initiatives.

Of course, other classifications and variables could be used to characterise energy blockchain initiatives, including (a) peer-reviewed publications, (b) active network nodes, and (c) periodic transaction value. However, project and research data does not exist to support the use of these particular variables. Therefore, classifying the ownership of each initiative's primary communication channel was used to derive general characteristics relating to technical transparency, project scale and commercialisation. Although claiming these characteristics can be determined by classifying communication channels alone would be unreasonable, the existence of dedicated channels (e.g., websites and blogs) demonstrate organisational and financial commitments that are synonymous with large-scale and commercial endeavours.

\subsection{RO4: Determine Depth of Technical Information}

Figure 5 illustrates the availability and depth of information shared across different types of energy blockchain initiatives. A simple scheme of low, moderate and high was used to classify the conceptual, theoretical and technical information shared, with low indicating that only superficial information could be accessed (e.g., press releases), and high indicating some useful and detailed information was available (e.g., whitepapers). The analysis shows that information sharing remains low across most types of energy blockchains, which naturally impedes efforts to align research agendas across academia and industry. Some potential reasons for low information sharing may include commercial sensitivity, technical instability, and regulatory concerns. 


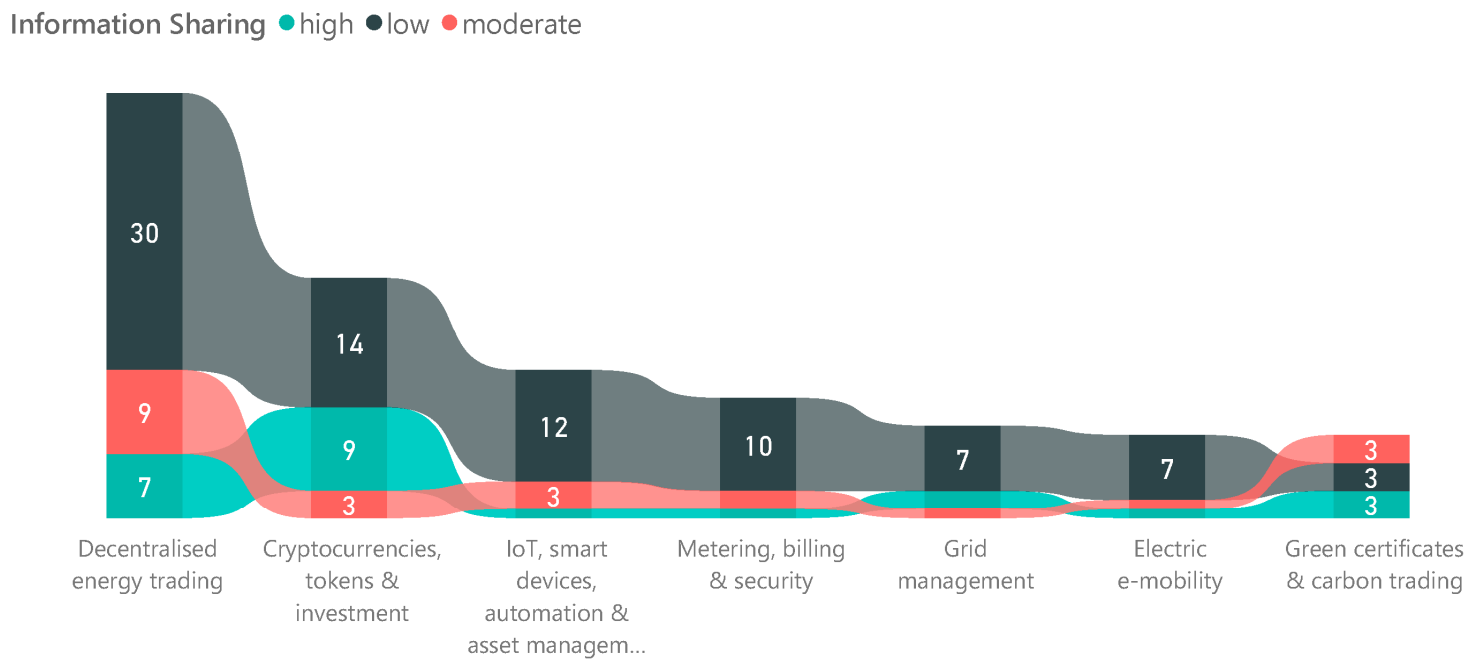

Figure 5. Level of information sharing by type of applications.

Agreeing and standardising the way initiatives were classified represented the most significant challenge for this particular analysis. A set of basic heuristics were created to aid the process, but some subjective decision-making was needed to appropriately classify edge cases. Generally, low information sharing indicated details were only available from press releases announcing the launch of the initiative, with no significant updates thereafter. Using the same stream of thought, moderate information sharing indicated updates were disseminated on more than one occasion post-launch (e.g., detailed blogs). Finally, high information sharing was largely reserved for initiatives with detailed whitepapers or substantial blog posts relating to either the real-world energy blockchain initiative, or the broader energy blockchain domain. Although not feasible due to the disparate and inconsistent nature of current information sharing protocols (i.e., initiatives share different types of details), lower-level classifications identifying the specific dimensions of information being shared (e.g., microgrid integration) would serve to better highlight challenges and opportunities, and provide the basis for an objective and well-defined research agenda.

\subsection{RO5: Determine Technical Trends}

A summary of technical trends extracted from the original dataset illustrate the prominence of classified energy applications (Figure 6), blockchain platforms (Figure 7), and consensus algorithms (Figure 8). Although these details are particularly useful for establishing the current approaches and trends, such trends should be regularly evaluated to ensure changes based on best-practices are captured. However, such longitudinal analysis greatly depends on accurate and accessible information being openly shared by grid operators and energy providers.

Figure 6 illustrates prominent energy blockchain applications, with decentralised energy trading and cryptocurrencies comprising almost $50 \%$ of energy blockchain initiatives. While the more popular applications centre on the democratisation of energy for individuals, groups and communities, the less popular applications (e.g., metering and billing) are more oriented towards organisations that may improve their ability to manage particular operating scenarios using blockchain technology (e.g., replacing centralised databases for metering). 


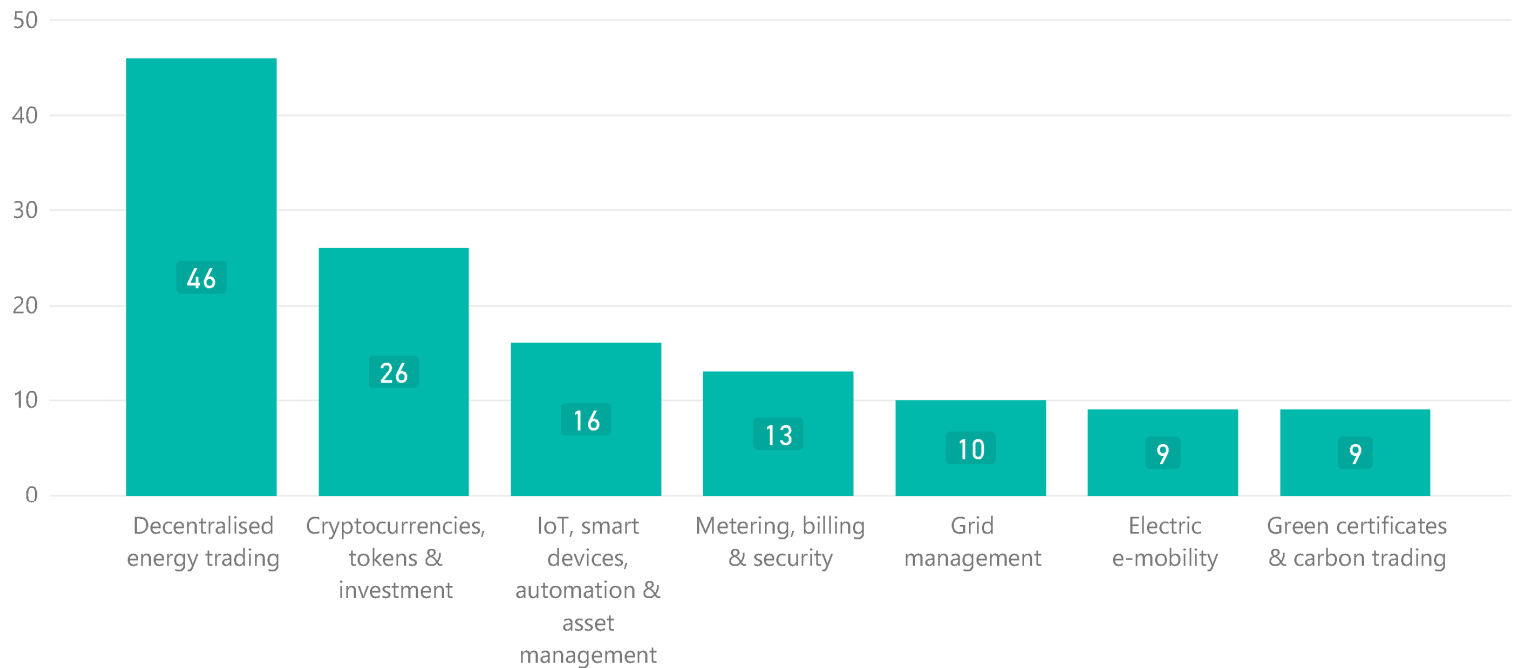

Figure 6. Classified energy blockchain applications.

Figure 7 illustrates the prominence of different blockchain platforms across energy blockchain initiatives. The analysis shows Ethereum as the most prominent platform (44.33\%), with Energy Web (12.37\%), Hyperledger Fabric (7.22\%) and Proprietary/Custom (7.22\%) platforms demonstrating significantly lower adoption.

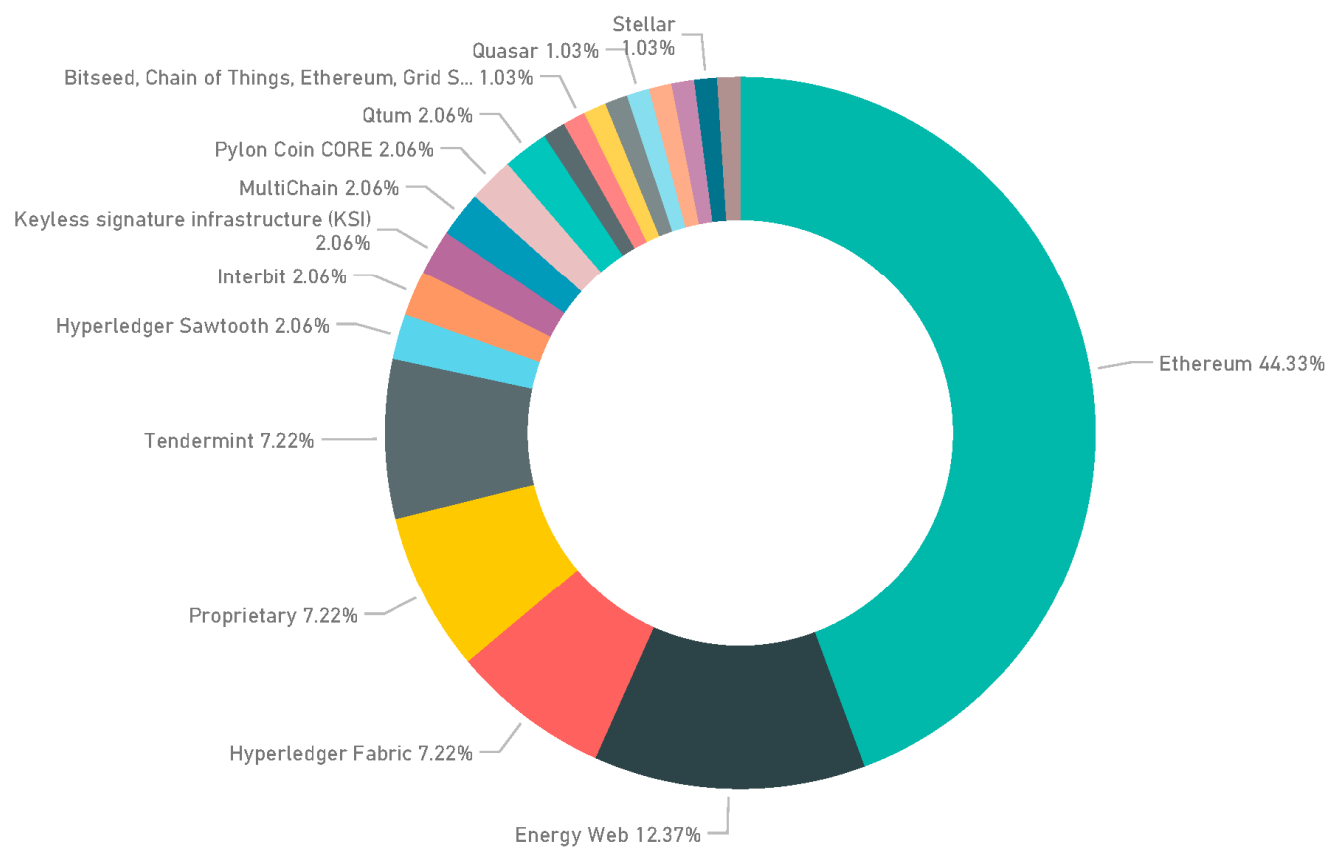

Figure 7. Blockchain platform adoption for energy blockchains.

Figure 8 illustrates the prevalence of consensus algorithms used to commit transactions to energy blockchains. Interestingly, despite well-known concerns regarding scalability and performance, the analysis highlights Proof-of-Work (49.4\%) as the most commonly used consensus algorithm, with Proof-of-Authority $(15.66 \%)$ and Practical Byzantine Fault Tolerance $(15.66 \%)$ demonstrating some signs of adoption. Of course, the adoption of particular consensus algorithms may be influenced by the contemporary nature of energy blockchains, whereby early adopters and researchers utilise the platform's default consensus model, or make choices derived from mainstream media (e.g., the Bitcoin phenomenon) that may not be directly applicable or useful to energy applications. However, provided 
sufficient information can be shared amongst practitioners and researchers, best-in-class methods and approaches shall invariably emerge to guide the selection of platforms and consensus algorithms for energy applications.

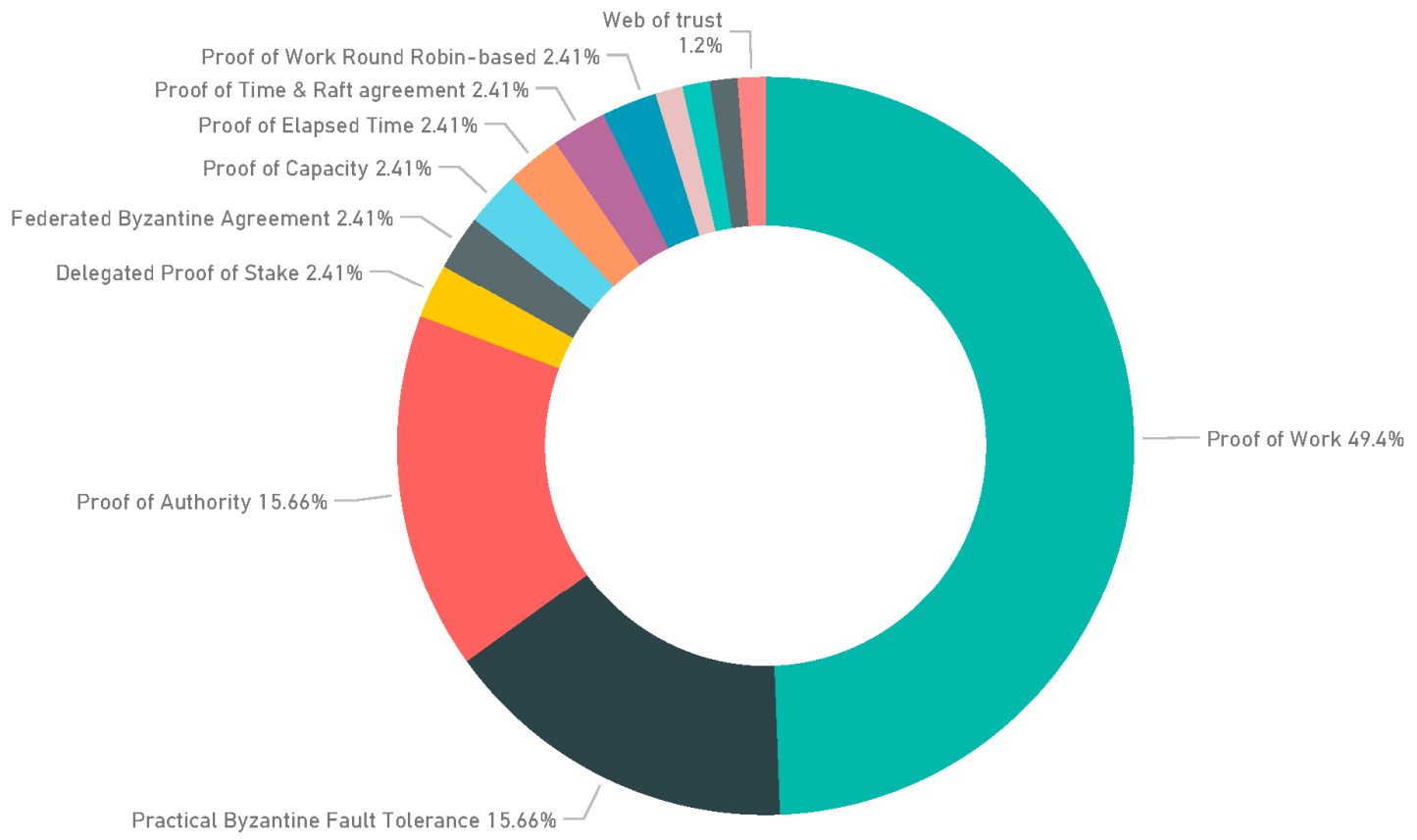

Figure 8. Consensus algorithm adoption for energy blockchains.

The trends relating to energy blockchain applications, blockchain platforms, and consensus algorithms are undoubtedly useful for developers and researchers investigating the technology within the energy sector, but inferring or developing best-practices for real-world energy blockchains cannot be easily achieved without additional information, such as (a) blockchain and infrastructure integration approaches (e.g., interdisciplinary protocols), (b) accounting methods for energy transactions (e.g., energy losses during transmission), and (c) disclosure of technical impediments (e.g., poor scalability or maintenance), to name a few.

\section{Conclusions}

Given the infrastructure and regulatory requirements surrounding the energy sector, commercial grid operators and energy providers are well-positioned to influence the rollout and direction of energy blockchains. However, privatising the development of real-world energy blockchains may discourage information sharing and open collaboration due to concerns regarding commercial sensitivity, competitiveness and intellectual property. Although these concerns are quite understandable from some perspectives, withholding technical or theoretical information serves to temper innovation and progress across the field, while increasing implementation costs for organisations given each must learn from their own mistakes, rather than learning from the broader body of knowledge. Indeed, sufficient information sharing and openness must exist for academic and industrial researchers to identify, explore and solve challenges that can genuinely contribute to the success of real-world implementations.

The overarching and fundamental aim of this research was to explore and understand the current state of real-world energy blockchains, which was addressed using a systematic and data-driven methodology comprising five core objectives. These objectives focused specifically on (RO1) determining growth rates and trends, (RO2) evaluating failure and drop-off rates, (RO3) identifying channels used for disseminating information, (RO4) determining theoretical and technical depth of information sharing, and (RO5) presenting fundamental trends for blockchain technologies, algorithms, tools and platforms. 
The analysis presented illustrates that real-world energy blockchains are growing exponentially year-on-year, while the majority of the identified energy blockchain initiatives formed between 2015 and 2018 remain active. Given the acceleration and growth of real-world energy blockchains, the development of standards and best-practices are urgently needed to circumvent the creation of isolated, disparate and proprietary energy blockchains that may prove difficult to reverse once deployed on physical grid infrastructure. However, establishing best-practices and coherent research agendas requires commercial and academic stakeholders to subscribe to information sharing protocols for the betterment of the general domain. Although the majority of real-world energy blockchains analysed served information to stakeholders through dedicated websites and other channels, the information disseminated through these channels did not possess the technical or theoretical depth needed to unify approaches, or contribute to the broader body of knowledge.

\section{Future Work}

Our future work shall focus on the establishment of a formal metadata specification and information exchange protocol for energy blockchains, which can facilitate the exchange of technical and theoretical details between organisations and researchers. In many respects, these future work items will attempt to bridge some of the information gaps and disconnects identified during the systematic analysis.

Author Contributions: P.O. established the research methodology, undertook data collection protocols, and compiled research results. D.T.J.O. managed the data classification process and compiled research results.

Funding: This research received no external funding.

Conflicts of Interest: The authors declare no conflict of interest.

\section{References}

1. Zizzo, G.; Sanseverino, E.R.; Ippolito, M.G.; Silvestre, M.L.D.; Gallo, P. A Technical Approach to P2P Energy Transactions in Microgrids. IEEE Trans. Ind. Inform. 2018.

2. Gürcan, Ö.; Agenis-Nevers, M.; Batany, Y.M.; Elmtiri, M.; Le Fevre, F.; Tucci-Piergiovanni, S. An Industrial Prototype of Trusted Energy Performance Contracts using Blockchain Technologies. In Proceedings of the 2018 IEEE 20th International Conference on High Performance Computing and Communications; IEEE 16th International Conference on Smart City; IEEE 4th International Conference on Data Science and Systems (HPCC/SmartCity/DSS), Exeter, UK, 28-30 June 2018.

3. Thomas, L.; Long, C.; Burnap, P.; Wu, J.; Jenkins, N. Automation of the supplier role in the GB power system using blockchain-based smart contracts. CIRED Open Access Proc. J. 2017, 2017, 2619-2623. [CrossRef]

4. Goranović, A.; Meisel, M.; Fotiadis, L.; Wilker, S.; Treytl, A.; Sauter, T. Blockchain applications in microgrids an overview of current projects and concepts. In Proceedings of the IECON 2017-43rd Annual Conference of the IEEE Industrial Electronics Society, Beijing, China, 29 October-1 November 2017; pp. 6153-6158.

5. Vangulick, D.; Cornélusse, B.; Ernst, D. Blockchain for peer-to-peer energy exchanges: Design and recommendations. In Proceedings of the 2018 Power Systems Computation Conference (PSCC), Dublin, Ireland, 11-15 June 2018.

6. Li, Y.; Yang, W.; He, P.; Chen, C.; Wang, X. Design and management of a distributed hybrid energy system through smart contract and blockchain. Appl. Energy 2019, 248, 390-405. [CrossRef]

7. Tan, S.; Wang, X.; Jiang, C. Privacy-Preserving Energy Scheduling for ESCOs Based on Energy Blockchain Network. Energies 2019, 12, 1530. [CrossRef]

8. Magnani, A.; Calderoni, L.; Palmieri, P. Feather forking as a positive force. In Proceedings of the 1st Workshop on Cryptocurrencies and Blockchains for Distributed Systems, Munich, Germany, 15 June 2018; pp. 99-104.

9. Pieroni, A.; Scarpato, N.; Di Nunzio, L.; Fallucchi, F.; Raso, M. Smarter City: Smart Energy Grid based on Blockchain Technology. Int. J. Adv. Sci. Eng. Inf. Technol. 2018, 298-306. [CrossRef]

10. Lombardi, F.; Aniello, L.; De Angelis, S.; Margheri, A.; Sassone, V. A blockchain-based infrastructure for reliable and cost-effective IoT-aided smart grids. In Proceedings of the Living in the Internet of Things: Cybersecurity of the IoT-2018, London, UK, 28-29 March 2018; pp. 1-6. 
11. Mylrea, M.; Gourisetti, S.N.G. Blockchain for smart grid resilience: Exchanging distributed energy at speed, scale and security. In Proceedings of the 2017 Resilience Week (RWS), Wilmington, DE, USA, 18-22 September 2017; pp. 18-23.

12. Wang, J.; Wang, Q.; Zhou, N.; Chi, Y. A Novel Electricity Transaction Mode of Microgrids Based on Blockchain and Continuous Double Auction. Energies 2017, 10, 1971. [CrossRef]

13. Pustišek, M.; Kos, A.; Sedlar, U. Blockchain Based Autonomous Selection of Electric Vehicle Charging Station. In Proceedings of the 2016 International Conference on Identification, Information and Knowledge in the Internet of Things (IIKI), Beijing, China, 20-21 October 2016; pp. 217-222.

14. Mengelkamp, E.; Notheisen, B.; Beer, C.; Dauer, D.; Weinhardt, C. A blockchain-based smart grid: Towards sustainable local energy markets. Comput. Sci. Res. Dev. 2017, 33, 207-214. [CrossRef]

15. Hwang, J.; Choi, M.-I.; Lee, T.; Jeon, S.; Kim, S.; Park, S.; Park, S. Energy Prosumer Business Model Using Blockchain System to Ensure Transparency and Safety. Energy Procedia 2017, 141, 194-198. [CrossRef]

16. Mengelkamp, E.; Gärttner, J.; Rock, K.; Kessler, S.; Orsini, L.; Weinhardt, C. Designing microgrid energy markets: A case study: The Brooklyn Microgrid. Appl. Energy 2018, 210, 870-880. [CrossRef]

17. Hukkinen, T.; Mattila, J.; Ilomäki, J.; Seppälä, T. A Blockchain Application in Energy; ETLA Reports: Helsinki, Finland, 2017; Volume 71.

18. Andoni, M.; Robu, V.; Flynn, D.; Abram, S.; Geach, D.; Jenkins, D.; McCallum, P.; Peacock, A. Blockchain technology in the energy sector: A systematic review of challenges and opportunities. Renew. Sustain. Energy Rev. 2019, 143-174. [CrossRef]

19. Yli-Huumo, J.; Ko, D.; Choi, S.; Park, S.; Smolander, K. Where Is Current Research on Blockchain Technology? A Systematic Review. PLoS ONE 2016, 11, e0163477. [CrossRef] [PubMed]

20. Conoscenti, M.; Vetro, A.; De Martin, J.C. Blockchain for the Internet of Things: A systematic literature review. In Proceedings of the 2016 IEEE/ACS 13th International Conference of Computer Systems and Applications (AICCSA), Agadir, Morocco, 29 November-2 December 2016.

(C) 2019 by the authors. Licensee MDPI, Basel, Switzerland. This article is an open access article distributed under the terms and conditions of the Creative Commons Attribution (CC BY) license (http://creativecommons.org/licenses/by/4.0/). 